\title{
Tako-Tsubo Phenomenon: Dual-Source Computed Tomography and Conventional Coronary Angiography
}

\author{
Hans Scheffel · Paul Stolzmann • Christoph Karlo $\cdot$ Pedro Trigo-Trindade • \\ Borut Marincek · Thomas F. Luescher · Hatem Alkadhi
}

Received: 15 June 2007/ Accepted: 27 June 2007/Published online: 4 August 2007

(C) Springer Science+Business Media, LLC 2007

\begin{abstract}
A 75-year-old woman was admitted to our emergency department with severe chest pain and mild dyspnea that started soon after a marital quarrel with her husband. Her 12-lead electrocardiogaphy (ECG) demonstrated an ST elevation in the precordial leads, and the plasma troponin $\mathrm{T}$ level was elevated to $0.29 \mathrm{ng} / \mathrm{mL}$. An acute myocardial infarction with ST elevation was assumed, and the patient was admitted for an emergency catheter coronary angiography. Her coronary arteries were normal, but left ventriculography demonstrated a severe systolic dysfunction involving the mid and apical myocardial segments, showing apical ballooning (Supplemental Data Movie I). Thus, the diagnosis of tako-tsubo phenomenon was made. The ejection fraction was decreased to $40 \%$. Because of a history of pulmonary embolism, the patient was referred for chest computed tomography (CT) to rule out a recurrent pulmonary embolism. Contrast-enhanced, retrospectively ECG-gated dual-source CT excluded pulmonary embolism and similarly demonstrated systolic dysfunction with apical ballooning (Supplemenal Data Movie II). After 2 days, the patient's symptoms ceased and echocardiography showed normalized left ventricular systolic function.
\end{abstract}

Tako-tsubo phenomenon is characterized by transient left ventricular systolic dysfunction of unknown etiology. Initially described in Japan [1] and named after an octopustrapping pot with a round bottom and a narrow neck, it has

H. Scheffel · P. Stolzmann - C. Karlo · B. Marincek ·

H. Alkadhi ( $\square)$

Institute of Diagnostic Radiology, University Hospital Zurich,

Raemistrasse 100, 8091 Zurich, Switzerland

e-mail: hatem.alkadhi@usz.ch

P. Trigo-Trindade - T. F. Luescher

Cardiovascular Center, University Hospital Zurich, Zurich,

Switzerland also been recognized more and more in Western countries $[2,3]$. It predominantly occurs in postmenopausal women and frequently after emotional stress. Importantly, such a psychological stressful trigger is not observed in all individuals, and its absence does not exclude the diagnosis. A few cases have been reported in younger women and males [4]. Rarely, patients present with syncope or an out-ofhospital cardiac arrest. Symptoms clinically mimic acute myocardial infarction, with the majority of patients presenting with chest pain, dyspnea, and mild ST-segment elevation in precordial leads followed by T-wave inversion involving most leads [2]. Plasma troponin $\mathrm{T}$ levels are either normal or only slightly increased and thus contrast the extent of systolic dysfunction in the acute phase. Patients are usually hemodynamically stable, but clinical findings of mild to moderate congestive heart failure commonly coexist. In a minority of patients, hypotension may occur from reduced stroke volume and occasionally from dynamic left ventricular outflow tract obstruction. Cardiogenic shock may be a rare complication. Patients with tako-tsubo phenomenon generally have a good prognosis in the absence of significant underlying comorbidity. The systolic dysfunction and the regional wall-motion abnormalities are transient and full recovery usually occurs in most cases without specific therapy $[2,3]$.

The initial imaging for patients with tako-tsubo phenomenon is usually performed with catheter coronary angiography because of the clinical suspicion of acute myocardial infarction. The left ventriculogram shows characteristic regional wall-motion abnormalities involving the mid and apical segments (Fig. 1). There is sparing of the basal systolic function, and the wall-motion abnormality extends beyond the distribution of any one single coronary artery. Apical sparing variants have also been described [5]. Echocardiography and magnetic resonance 
Fig. 1 Catheter angiography of the left ventricle (A, B).

Ventriculogram of the diastolic (A) and systolic (B) phase in

$30^{\circ}$ right anterior oblique projection. Systolic phase revealed dysfunction of the left ventricular myocardium with hypercontractility of the basal parts (arrows) and reduced myocardial wall motion of the mid to apical regions leading to apical ballooning during systole (arrowheads). Dual-source computed tomography of the left ventricular myocardium $(\mathbf{C}$, D). Thick maximum intensity projections of the diastolic (C) and systolic (D) phase of the cardiac cycle in $30^{\circ}$ right anterior oblique plane resembling the left ventriculogram in demonstrating apical ballooning during systole (arrowheads) accompanied by hypercontractility of the basal myocardium (arrows)
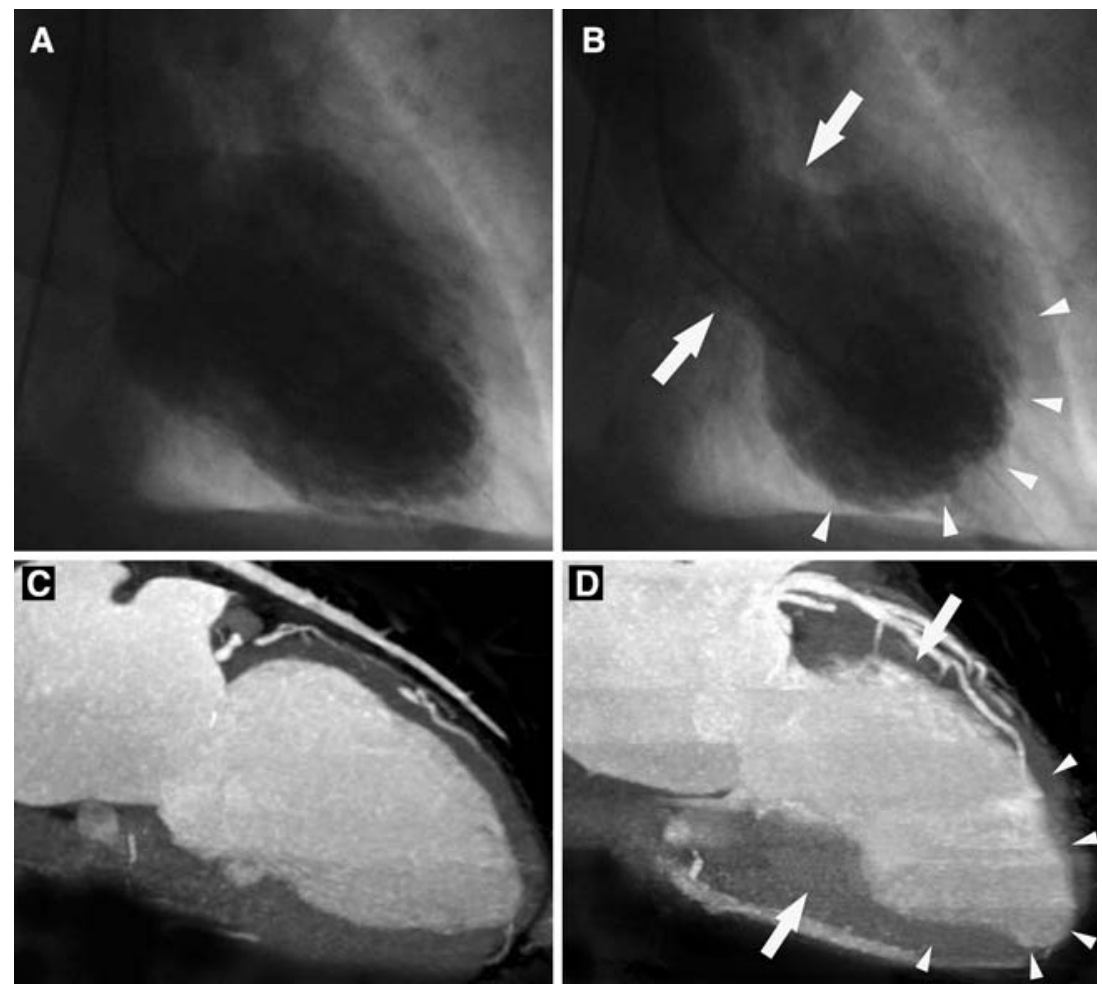

imaging have been shown to be excellent both for diagnosis and for monitoring restitution of left ventricular function. CT usually does not represent the first-line imaging modality for this disease but, similarly, allows the detection of regional wall-motion abnormalities indicating apical ballooning, as shown in this case. Due to the increasing use of CT for emergency evaluation of patients with acute chest pain, recognition of this rare entity may become important, particularly in patients with equivocal clinical and laboratory findings.

\section{References}

1. Dote K, Sato H, Tateishi H, et al. (1991) [Myocardial stunning due to simultaneous multivessel coronary spasms: a review of 5 cases]. J Cardiol 21(2):203-214
2. Prasad A (2007) Apical ballooning syndrome: an important differential diagnosis of acute myocardial infarction. Circulation 115(5):e56-e59

3. Stollberger C, Finsterer J, Schneider B (2006) Transient left ventricular dysfunction (tako-tsubo phenomenon): findings and potential pathophysiological mechanisms. Can J Cardiol 22(12): 1063-1068

4. Bybee KA, Kara T, Prasad A, et al. (2004) Systematic review: transient left ventricular apical ballooning: a syndrome that mimics ST-segment elevation myocardial infarction. Ann Intern Med 141(11):858-865

5. Hurst RT, Askew JW, Reuss CS, et al. (2006) Transient midventricular ballooning syndrome: a new variant. J Am Coll Cardiol 48(3):579-583 